\title{
DERECHO DE LAS MADRES TRABAJADORAS EN MÉXICO
}

\section{Lic. José Manuel Salvador Hernández ${ }^{1}$ \\ Dr. José Antonio Morales Notario ${ }^{2}$}

Resumen: El trabajo está reconocido como un derecho humano, inherente a las personas, por lo cual el Estado está obligado a garantizar las mejores condiciones, a fin de que todas las personas puedan ejercerlo en igualdad de circunstancias y libre de discriminación. Las mujeres se han incorporado de manera importante al mercado laboral y un número importante ya se percibe como cabeza de familia o como sostén del hogar, pero aún en condiciones desiguales con respecto a los hombres.

Un aspecto importante es la inclusión de la mujer en el espacio productivo, se están abriendo espacios en todas las áreas laborales de manera lenta; sin embargo, en el hogar, al contrario, los hombres siguen manejando una participación insignificante, lo que propicia condiciones de desigualdad.

Si bien es cierto, en la ley se establece la igualdad de género por lo que la mujer al momento de embarazarse debe contar con mejores condiciones de desarrollo y, por equidad, es necesario que se le proteja desde el momento de la concepción. embarazada, derechos laborales

\begin{abstract}
Work is recognized as a human right, inherent in people, for which the State is obliged to guarantee the best conditions, so that all people can exercise it under equal circumstances and free of discrimination. Women have entered the labor market in an important way and a significant number are already perceived as head of the family or as breadwinners, but still in unequal conditions with respect to men. An important aspect is the inclusion of women in the productive area, spaces are being released in a slow manner; however, at home, on the contrary, men continue to manage a negligible participation, which leads to unequal conditions in the task division.
\end{abstract}

Palabras-claves: Trabajo, mujer

\footnotetext{
${ }^{1}$ Licenciado en derecho por la Universidad Juárez Autónoma de Tabasco

${ }^{2}$ Doctor en Derecho. Profesor Investigador de Tiempo completo en la División Académica de Ciencias Sociales y Humanidades de la Universidad Juárez Autónoma de Tabasco
} 
While the law establishes gender equality, women, at the time of pregnancy, must have better conditions of development and, for equity, it is necessary to be protected from the moment of conception

Keywords: Work, pregnant woman, labor rights

\section{Introducción}

El marco jurídico nacional conformado por la Constitución Política de los Estados Unidos Mexicanos, la Ley Federal del Trabajo y la Ley General para la Igualdad para las Mujeres y Hombres reconocen los derechos laborales de las mujeres y, en el ámbito internacional el Estado Mexicano ha ratificado ente otros, el Convenio 100 sobre igualdad de remuneración, el Convenio 111 sobre la discriminación y la Convención para la eliminación de todas las formas de discriminación contra la mujer (CEDAW).

El efectivo goce de los derechos laborales de las mujeres es un requisito indispensable para el desarrollo en condiciones de igualdad en la esfera laboral. Sabemos por la experiencia vivida, que entre hombre y la mujer existe diferencias muy claras: las biológicas, físicas, psicológicas, entre otras; pero también es cierto que tienen grandes similitudes y, en la actualidad, dichas similitudes y diferencias se encuentran establecidas en nuestra Constitución Política de los Estados Unidos Mexicanos y en la Ley Federal del Trabajo, en las cuales señalan que tanto el varón como la mujer tienen iguales derechos.

La legislación jurídica laboral en México ha creado una serie de medidas dirigidas a proteger a la mujer en estado de gravidez, encontrando su regulación jurídica en los artículos 164 al 172 de la legislación laboral (Diputados, 2018). Proteger a la mujer debe ser esencial para cualquier gobierno y un primer caso que puede darse es que como futura mamá teman confesar haber quedado embarazada en un trabajo y principalmente si llevan poco tiempo, por miedo a ser despedidas.

Sin embargo, aunque esta situación les ha ocurrido a muchas mujeres y se sientan incómodas al dar las noticias, lo cierto es que su condición no puede utilizarse legalmente en su contra debido a la protección legal con la que cuentan en la legislación laboral. 
En primer lugar, es ilegal que el patrón las trate de forma menos favorable debido al embarazo, y eso es así desde el momento en el que la empleada sabe que está embarazada, sin importar si la madre trabajadora lleva mucho o poco tiempo prestando sus servicios en el centro de trabajo.

$\mathrm{Y}$ es que el embarazo, o la simple posibilidad de que la mujer en edad reproductiva lo esté, genera una de las manifestaciones más frecuentes de discriminación. Es necesario construir y fortalecer los marcos jurídicos para que cuenten con protección, así como contar con mecanismos que les garanticen el cumplimiento de la ley. Es necesario promover la participación de la mujer en el ámbito laboral en igualdad de condiciones con los hombres.

La protección durante el embarazo y la maternidad responde a una doble finalidad: proteger la especial relación entre la madre y el recién nacido y la salud de ambos, y brindar una cierta seguridad en el empleo (posibilidades de acceso al empleo para las mujeres en edad de concebir, mantenimiento de los salarios y prestaciones durante la maternidad y prevención de los despidos) (OIT, 2009).

\section{7}

\section{Legislación En Materia De Derechos}

\section{De Las Madres Trabajadoras}

Es conveniente aclarar que, aun cuando el artículo 4 de la Constitución Política de los Estados Unidos Mexicanos (Diputados, 2018) y diversos artículos de la Ley Federal del Trabajo (Lobato, 2013), establecen la igualdad sustantiva entre el hombre y mujeres, es imposible de conseguir la igualdad absoluta entre ambos sexos. Por principio, sólo las mujeres pueden embarazarse y eso genera una condición especial que merece romper con cualquier principio de igualdad.

De hecho, el Título Quinto de la Ley Federal del Trabajo (Diputados, 2018) se denomina "Trabajo de las mujeres", pero básicamente se orienta a establecer condiciones especiales que protejan a la mujer embarazada o, en su caso, a la mujer que, además de tener la característica de trabajadora, es madre.

El artículo 164 de la Ley Federal del Trabajo señala: "Las mujeres disfrutan de los mismos derechos $\mathrm{y}$ tienes las mismas obligaciones que los hombres" (Diputados, 2018). En otras palabras, la simple condición del sexo de un trabajador o trabajadora no genera condiciones especiales. Varones y 
mujeres disfrutan de derechos iguales y están obligados en igual medida. Lo que si genera diferencia en las condiciones es el embarazo o la condición de ser madre.

El Título Quinto de la Ley laboral no es, en ninguna medida violatoria de este principio de igualdad; simplemente es un reconocimiento de una situación especial (el embarazo o ser madre) que solo puede darse en las mujeres y que significa la aplicación de condiciones especiales que conlleva una protección mayor (Diputados, 2018).

Es importante mencionar que la diferencia entre el trabajo de hombres y mujeres en el trabajo, es la condición de embarazo.

$$
\begin{aligned}
& \text { de esta forma, si una mujer, sin } \\
& \text { estar embarazada y sin ser } \\
& \text { madre, trabaja, estará sujeta a } \\
& \text { las mismas obligaciones y } \\
& \text { tendrá los mismos derechos que } \\
& \text { sus compañeros varones. si en } \\
& \text { el desarrollo de la relación de } \\
& \text { trabajo se embaraza, } \\
& \text { comenzará a recibir estos } \\
& \text { beneficios especiales que se } \\
& \text { prolongarán hasta después del } \\
& \text { parto (Mendez, 2014). }
\end{aligned}
$$

De ahí que se desprenda que las condiciones establecidas para las madres trabajadoras no son discriminatorias, toda vez que las condiciones especiales durarán única y exclusivamente durante el estado de gestación y lactancia.

\section{Condiciones Especiales Para Mujeres}

\section{Embarazadas En México}

Las mujeres embarazadas $\mathbf{0}$ madres trabajadoras, cuentan con condiciones especiales y la Ley reconoce ese derecho particular que tienen en relación a los hombres, por ello, su fundamento constitucional de la fracción 5 apartado A del artículo 123 constitucional (Diputados, 2018) señala: Las mujeres durante el embarazo no realizaran trabajos que exijan un esfuerzo considerable $y$ signifiquen un peligro para su salud en relación con la gestación; gozará forzosamente de un descanso de seis semanas anteriores a la fecha fijada aproximadamente para el parto y seis semanas posteriores al mismo, debiendo percibir su salario íntegro y conservar su empleo y los derechos que hubiera adquirido por la relación de trabajo. En el período de lactación tendrán dos descansos extraordinarios por día de 
media hora cada uno para alimentar a sus hijos. ${ }^{3}$

Primeramente, el artículo 164 de la ley laboral establece la igualdad entre hombre y mujeres. La existencia de este artículo no es, por ningún motivo discriminatorio o violatorio del principio de igualdad; por ello, ese numeral señala que: "las mujeres disfrutan de los mismos derechos y tiene las mismas obligaciones que los hombres".

Por ello, es claro que estamos ante el principio de igualdad entre hombre y mujeres; y el hecho de ser fémina, no significa que deba tener un trato diferente a los varones, esto es, deberán desempeñar un cargo o funciones en el centro de trabajo $\sin$ importar su género.

Sin embargo, nuestra legislación laboral, hace una distinción al momento de abordar el tema de la madre trabajadora, pues en ésta se establece que el propósito fundamental es la defensa de la maternidad. De esta forma, se enfoca en la protección única y exclusivamente a las madres trabajadoras.

De ahí que, no todas las mujeres trabajadoras puedan gozar con el trato

3 Este precepto constitucional podemos encontrarlo de forma detallada en el titulo quinto de la citada Ley Federal del Trabajo, denominado especial; sólo aquella que se encuentra en estado de gestación o en periodo de lactancia. Nuestra legislación hace hincapié a una seria de prohibiciones que éstas tienen durante este periodo, las cuales son las que se enuncian a continuación:

- no pueden realizar labores insalubres o peligrosas.

- no pueden desempeñar trabajos nocturnos industriales alguno.

- Tampoco pueden laborar en establecimientos comerciales o de servicios después de las diez de la noche.

- No pueden trabajar horas extras.

Esas limitantes no significan que las mujeres sufran perjuicios en salarios, prestaciones o derechos. La Ley Federal del Trabajo también reconoce como labores peligrosas o insalubres las que por la naturaleza del trabajo, por las condiciones físicas, químicas $\mathrm{y}$ biológicas del medio que se presentan, o por la composición de la materia prima que se utilicen, son capaces de actuar sobre la vida y la salud física y mental de la mujer en estado de gestación, o del

"Trabajo de las mujeres", que comprende de los artículos 164 al 172. 
producto; es decir, las actividades, que por el lugar en el que se realicen o por la materia que se ocupen pongan en riesgo la salud tanto de la madre como del producto (Mendez, 2014).

Ahora bien, el artículo 170 de la Ley Federal del Trabajo fija los derechos de las madres trabajadoras:

- Durante el embarazo no realizarán trabajos que requieran un esfuerzo considerable $\mathrm{y}$ representen un peligro para su salud, lo que incluye tareas de levantar, tirar o empujar grandes pesos; que provoquen trepidación (temblor) o alteración en el estado psíquicos o nervioso, o estar de pie por tiempo prolongado.

- Descansos de seis semanas antes y seis semanas después de la fecha tentativa del parto. Aun cuando el precepto legal resulta claro en este aspecto, el mismo permite que cuatro de las seis semanas de descansos previas se trasfiera para después del parto. En otras palabras, una trabajadora podría gozar de dos semanas previas al parto y diez posteriores al mismo. Las condiciones que se fijan para que proceda esta modificación es que lo solicite expresamente la trabajadora y lo autorice algún médico de la institución de seguridad social o del servicio de salud que otorgue el patrón. Se tomará en cuenta la opinión del patrón y del trabajo que se desempeña.

- Se permite a la trabajadora hasta ocho semanas después del parto si los hijos nacen con alguna discapacidad o requieran atención médica hospitalaria. Es decir, una trabajadora puede disfrutar de dos semanas previas y hasta doce posteriores al parto, atendiendo las condiciones referidas.

- Un derecho incluido recientemente al cuerpo de la Ley Federal del Trabajo es el goce de seis semanas de descanso en caso de adopción. Estas semanas serán posteriores al día en que reciba al niño o niña en adopción.

- El descanso de las seis semanas previas y posteriores al parto pueden prolongarse por el tiempo necesario cuando la mujer esté 
imposibilitada para trabajar a causa del embarazo o del parto.

- Además del descaso diario de media hora, dispone también de dos descansos extraordinarios, de media hora cada uno, para alimentar a sus hijos en un lugar higiénico que la empresa designe. Este periodo de lactancia tiene un término máximo de seis meses. En la práctica laboral, los descansos de media hora se acumulaban y se recortaba una hora la duración de la jornada de trabajo, práctica que ahora reconoce y reglamente la ley.

- El salario dentro de las doce semanas obligatorios se les paga completo. $\mathrm{Si}$ el tiempo se prorroga, la trabajadora tiene derecho a recibir $50 \%$ de su salario, hasta por un periodo de sesenta días. No se especifica que sucede después de los sesenta días, pero se entiende que el patrón queda exento del pago del salario.

- El puesto que ocupaba al momento de retirarse por el parto se conservará hasta por un año; es decir, si la mujer después del parto decide regresar a su trabajo, conservará el mismo con las mismas condiciones y en la misma posición laboral.

- La trabajadora no pierde su antigüedad por el parto, al contrario, tiene derecho a que se le compute los periodo prenatales y posnatales.

Es importante resaltar que la Ley Laboral reconoce la necesidad de que se creen guarderías infantiles de las madres trabajadoras. Estos servicios son prestados por el Instituto Mexicano del Seguro Social, conforme a las propias disposiciones del instituto.

\section{Derecho De Las Madres Trabajadoras}

Dentro de una situación concreta y más aún dentro de una problemática determinada como es el caso de la mujer trabajadora en actualidad, las interrogantes surgen con respecto a cuáles son las circunstancias desfavorables con las que cada mujer tiene que lidiar diariamente para poder desempeñarse adecuadamente en su rol de madre y al mismo tiempo tener un adecuado desempeño laboral. 
Se considera que el papel de madre por sí mismo, es un papel de mucha dedicación, paciencia, etc., aunado con el hecho de que la mujer trabaje, en una primera instancia como una necesidad económica de los tiempos actuales, para poder ajustar los gastos del hogar; y por otra parte para seguir desarrollándose profesionalmente con la misma equidad de género que nuestros días se demanda. Ambas circunstancias han hecho muy complejo este doble rol de la mujer en la actualidad.

Dentro de las distintas ideas que se mencionan sobre los derechos de la mujer en relación a su situación de madre trabajadora, Héctor Santos Azuela (1998) señala los siguientes:

"El sistema se
encuentra orientado,
desde el punto de vista
laboral, a igualar su
calidad profesional
frente a los hombres,
disfrutando de
idénticos derechos y
condiciones generales
de trabajo.
$\ldots$ Atento a esta
circunstancia, el
legislador previene que
las mujeres de los
mismos derechos, a la
vez que cuentan con
las mismas
obligaciones laborales
que los hombres
(Artículo 164 de la Ley
Federal del Trabajo).

En este sentido, se ha apuntado que el embarazo, la maternidad y el parto constituyen la razón del trato jurídico diferenciado para la mujer trabajadora.

El régimen vigente se ciñe al Convenio 161 de la OIT respecto al trabajo de mujeres protegido, en atención al siguiente criterio:
- Evitar su utilización en labores de trituración $\quad 0$ molienda de materia en transformación.

- No emplearlo en empresas

dedicadas a la ingeniería civil, que reportan alto riesgo.

- No utilizarlo tampoco para el trabajo ferroviario, ni en la construcción de Buques.

- Así como evitar contratarlo para la producción, transformación $\mathrm{y}$ transmisión de energía eléctrica o cualquier tipo de fuerza motriz.

El Legislador prevé la situación de la mujer, como condición fundamental, el evitar que la actividad laboral ponga en peligro la salud de la mujer o del producto, bien sea durante el Estado de gestación o en aquel de 
lactancia (artículo 166 de la Ley Federal del Trabajo).

A tal efecto se previene que las mujeres no habrán de realizar trabajos físicos fuertes que impliquen esfuerzos materiales excesivos durante los tres meses que antecedan al parto (artículo 170, fracción I de la Ley Federal del Trabajo)."

Ahora bien, las principales necesidades de la madre trabajadora, que en primera instancia tendrían que ser garantizadas por el Estado y el patrón en su caso:

- Mujeres Iguales

- Derechos y obligaciones

- Protección a la maternidad

- Servicio de Guardería

Otra explicación más sobre los derechos de la madre trabajadora en nuestro país, lo señala Patricia Kurczyn (2000) cuando señala que la maternidad es un hecho jurídico que está relacionado con la reproducción del ser humano. Por ello, surgen derechos y obligaciones. Las mujeres que prestan sus servicios como empleadas, tiene derechos que están relacionados con su condición de maternidad; por ello, la legislación laboral y la de seguridad social contienen disposiciones que las protegen, pues ven a esta condición de la maternidad, como un asunto de salud, también regulado por las legislaciones sanitarias.

La maternidad, es una función reproductiva de la mujer que comprende la gestación y el embarazo. La ley general de salud considera, dentro de la maternidad, al embarazo, parto $\mathrm{y}$ puerperios. Esto indica que, si bien es cierto, tanto la mujer como el hombre son fisiológicamente diferentes, son iguales como seres humanos. El reto de la igualdad jurídica radica principalmente en el reconocimiento que se haga sobre esta diferencia y tratar a la mujer con las mismas consideraciones y respeto que el varón; fijando principal atención a la maternidad y el tiempo de procreación.

Esta protección es necesaria, principalmente porque toda persona tiene derecho a la salud y, de igual forma, la ley protege la organización y desarrollo de la familia. De igual forma, la maternidad es una función social vital que permite la continuación de la descendencia humana.

Por ello, la limitación, restricción o privación de los derechos laborales que sufren las mujeres con 
motivo de la maternidad y la falta de oportunidades para el cuidado de su salud y la de su hija o hijo, constituye una vulneración a su condición de madre. Es necesario proteger a la trabajadora durante la gestación, embarazo, parto, puerperio y lactancia, aunado a que los derechos de maternidad son prestaciones que ellas tienen por el hecho de prestar un servicio remunerado.

Con motivo de la gestación, las atenciones deben abarcar:

- cuidar su condición fisiológica;

- cuidar el desarrollo del producto de la concepción;

- contar con atención médica especializada para ella y para su hijo aún no nacido;

- conservar su puesto de trabajo con la remuneración correspondiente y todos los derechos inherentes al mismo, $\mathrm{y}$

- disfrutar de descansos para atender el alumbramiento y para cuidar al recién nacido.
Por su parte los patrones no pueden:

- rechazar a una solicitante de un trabajo por estar embarazada, no promoverla, o despedirla por ese motivo;

- excluirla de los beneficios, promociones, ascensos, capacitación y adiestramiento, por su embarazo;

- exigirle la renuncia a causa del embarazo, puerperio, lactancia o responsabilidades familiares, o

- disminuir cualquiera de sus derechos laborales o de seguridad social.

Estas prohibiciones, como se puede advertir, coinciden con factores de discriminación. Los derechos se producen antes, durante y después del embarazo.

Antes del Embarazo:

- La mujer tiene derecho a ser informada sobre los métodos anticonceptivos y atendida para evitar el embarazo cuando así lo 
solicite. Recuérdese que las personas son libres para decidir responsablemente sobre el número de hijos que desea procrear.

Durante el embarazo:

- Debe contar con asistencia médica, proporcionada a través de especialistas, medicinas y hospitalización en caso necesario.

- No debe realizar labores insalubres o peligrosas, ni trabajo nocturno industrial.

- No debe ocuparse en jornadas después de las diez de la noche en el establecimiento comercial o de servicio.

- No debe desempeñar horas extraordinarias.

- Tiene derecho a descansar durante seis semanas anteriores a la fecha del parto, con el disfrute de su salario íntegro.
- En caso de requerirlo, ese descanso puede prorrogarse hasta por sesenta días, percibiendo el $50 \%$ de su salario.

Después del embarazo:

- Debe disfrutar del descanso postnatal, por lo menos durante seis semanas después del parto.

- Disfrutar de la remuneración íntegra durante ese descanso.

- Cuando las necesidades de salud de ella o del recién nacido exijan un tiempo mayor de descanso podrá disfrutarlo hasta por sesenta días después de las seis semanas después del parto, recibiendo, el $50 \%$ de su salario.

- Conservará su puesto de trabajo, hasta por un año después de la fecha del parto sin goce de salario.

- Disfrutará de algunas prerrogativas para la alimentación y el cuidado 
de su hijo o hija. La Ley otorgara el derecho a dos descansos de media hora cada uno, durante la jornada de trabajo, para alimentarlo.

- Durante el periodo en que la trabajadora este alimentando a su hijo o hija no deberá realizar labores cuando se exponga a sustancias químicas que pueden afectar su salud o la de hijo o hija.

Kurczyn (Kurczyn, 2000) señala que hay que considerar otras posibilidades, tales como la fecha en que la mujer trabajadora puede pedir su incapacidad, o en relación a la hora de lactancia. Ante ello, las mujeres trabajadoras deben negociar con el patrón el momento en que tomarán su descanso prenatal para juntar los días o las semanas con la licencia después del parto. Sin embargo, el patrón no está obligado a aceptarlo, y en determinado momento, será el médico responsable de la atención de la mujer, el que lo autorice para evitar daños en la salud de la madre o del producto.
96

Si bien es cierto, estos permisos se pueden pactar previamente al momento de la celebración de los contratos de trabajo, la ley permite que se puedan cambiar, siempre y cuando las normas favorezcan a las trabajadoras. En caso de existir un arreglo para acumular los permites, es importante que se haga por escrito para protección y seguridad de las partes. De esta forma podrán evitar descuentos, sanciones y hasta que sean despedidas de manera injustificada.

La licencia médica que se expide con el parto, es fijada por el médico que trata a la madre trabajadora. Sin embargo, ante la posibilidad de que la fecha sea imprecisa, se debe considerar la posibilidad de ajustarla, de acuerdo a las necesidades biológicas de la madre trabajadora.

La Ley del Seguro Social reconoce que la maternidad inicia a partir del día en que el médico de la institución, certifica el embarazo. Pero, en aquellos casos en que la trabajadora no está asegurada y que no exista otro servicio médico proporcionado por el patrón, éste debe admitir el certificado del médico que la trabajadora le presenta.

En cuanto a las actividades peligrosas o insalubres que ponen en riesgo la salud de la mujer embarazada, 
el artículo 167 de la Ley Federal del Trabajo determina estas actividades de manera general, y las ubica por la naturaleza misma del trabajo que se desempeña, así como por las condiciones físicas, químicas o biológicas del medio en que se presta el trabajo o por la materia prima que se maneje; debido a que son capaces de actuar sobre la vida y la salud física y mental de la mujer en estado de gestación o del producto.

De igual manera, la ley contempla el cambio temporal de las actividades que normalmente desempeña en caso de que el patrón, al conocer el estado de la gestación, no le asigne otras funciones para. Después del nacimiento o de la lactancia, la trabajadora deberá volver a sus actividades acostumbradas y lugar habitual.

Dentro de este contexto, éstos derechos se encuentran regulados, pero distan mucho de poderse implementar en México, principalmente por la falta de conciencia sobre la importancia que debe dársele a la mujer, así como a sus derechos establecidos constitucionalmente, tanto en el ámbito de su desarrollo como madre, así como el de poder desempeñarse profesionalmente o simplemente poder seguir en la vida laboral, y que en muchos por desgracia es por una imperiosa necesidad económica.

José Dávalos (2000) insta a revalorizar el trabajo de las mujeres e indica que el derecho al voto de 1953 y la igualdad jurídica del hombre frente a la ley de 1974, son las reivindicaciones jurídicas más importantes de la mujer mexicana en el presente siglo.

En ese sentido, en la exposición de motivos de la reforma constitucional de 1974 se estableció que:

“...la única diferencia que puede establecerse válidamente entre los derechos de la mujer y el hombre, será aquella que se derive de la protección social de la maternidad, preservando la salud de la mujer y del producto en los periodos de gestación y lactancia..."....

Si bien es cierto, existen las mujeres enfrentan diversos problemas para incorporarse al mercado de trabajo, tales como la negativa patronal a contratarlas si son casadas o si están embarazadas; el despido del trabajo si se embarazan; el acoso sexual; un catálogo de posibilidades de empleo mucha más 
participación de la mujer en las labores productivas, etc., debe tomarse en cuenta que si se desea mejorar la situación de la mujer trabajadora, es necesaria la reestructuración de los descansos para alimentar a los hijos; flexibilizarse los periodos pre y postnatales y permitir el ingreso a la vida sindical, a las mujeres sobre todo a las jóvenes.

En la exposición de motivos se propone cambiar la reducción temporal de la jornada (dos horas diarias), al inicio o al final de la jornada, para brindar cuidados a sus hijos, en vez de los actuales dos periodos de descanso, de media hora cada uno que se otorgan para alimentar a sus hijos.

En ese mismo sentido, es conveniente cambiar el actual espacio de tres meses por el descanso pre $\mathrm{y}$ postnatal de seis meses; que la madre trabajadora lo disfrute distribuyéndolo en la forma que más le convenga con la autorización del médico que la atiende. Y, si la mujer deja de participar en algunas actividades productivas que requieren de considerables esfuerzos físicos por cuestiones biológicas, esto no debe ser impedimento para que asciendan a los cargos directivos.

El trabajo femenino debe revalorizarse y darse con base en que las madres trabajadoras son una inversión, de la que habrán de surgir, buenos y abundantes frutos sociales.

"El artículo 165 de la Ley determina que las modalidades de las normas aplicables al trabajo de las mujeres, tienen como propósito fundamental la protección de la maternidad. Sin que la madre sufra perjuicio en su salario, prestaciones y derechos (artículo 166), durante el estado de gestación y la lactancia, no se puede utilizar su trabajo en labores insalubres y peligrosas, en trabajo nocturno industrial." La mujer es discriminada desde antes de ser admitida en un trabajo. Se le pide como requisito de ingreso un certificado de ingravidez. Se le somete a interrogatorios orientados a poner al descubierto si es su intención embarazarse $o$ casarse a corto plazo. De acuerdo a los resultados se les otorga o no el empleo. Tal parece que quienes ponen estas trabas a las mujeres hubieran nacido en un envase de vidrio y no del vientre de una madre" (Lechuga, 2016).

\section{Conclusión}

Es así como a grandes rasgos puede observarse que a pesar de existe una legislación que protege algunos 
derechos de la mujer, en la realidad quedan aún por resolverse muchas situaciones injustas para las madres trabajadoras.

De igual forma se considera que en muchos aspectos podrían ser fortalecidos los derechos ya existentes con el propósito de lograr una eficaz forma de protección tanto a la madre, como al recién nacido, además de existir otros casos que ni siquiera están contemplados como son los casos de adopción o los derechos de una nueva paternidad, como puede ser en el caso de los padres trabajadores, permitiendo así que la mujer pueda tener mayor apoyo, y así poder reincorporarse en la vida laboral más pronto $\mathrm{y}$ en mejores condiciones.

\section{Bibliografía}

Dávalos, J. (2000). Tópicos Laborales. México: Porrúa.

Diputados. (27 de 08 de 2018). Constitución Política de los Estados Unidos Mexicanos. Obtenido de Cámara de Diputados del H. Congreso de la Unión. Secretaría General: |http://www.diputados.gob.mx/LeyesBi blio/pdf/1_270818.pdf
Diputados. (22 de 06 de 2018). Ley Federal del Trabajo . Obtenido de Cámara de Diputados del H. Congreso de la Unión. Secretaría General: http://www.diputados.gob.mx/LeyesBib lio/pdf/125_220618.pdf

Kurczyn, P. (2000). Derecho de las madres trabajadoras. México: UNAM.

Lechuga, E. (2016). Constitución Política de los Estados Unidos Mexicanos. México: Ediciones fiscales ISEF.

Lobato, J. (2013). Nueva Ley Federal del Trabajo. México: Barbera Editores.

Mendez, R. (2014). Derecho Laboral: un enfoque práctico. México: Mc Graw Hill.

OIT. (08 de mayo de 2009). Informe VI: La igualdad de género como eje del trabajo decente. Obtenido de Organización Internacional del Trabajo: https://www.ilo.org/ilc/ILCSessions/98t hSession/ReportssubmittedtotheConfere nce/WCMS_106175/lang--es/index.htm

Santos, H. (1998). Derecho del Trabajo. México: Mc Graw Hill. 